\title{
SINGULAR MULTIPLE INTEGRALS, WITH APPLICATIONS TO
}

\section{SERIES*}

BY

\section{BURTON H. CAMP}

Introduction.

The object of this paper is the study of the convergence to zero of Lebesgue integrals of the type

$$
\int_{E} f(t, u, \cdots) \phi(t, u, \cdots ; n, m, \cdots) d E,
$$

and to $f(x, y, \cdots)$ of singular Lebesgue integrals of the type

$$
\int_{E} f(t, u, \cdots) \theta(t, u, \cdots ; x, y, \cdots ; n, m, \cdots) d E,
$$

where $E$ is a limited, measurable field in space of $i$ dimensions, $f$ is a function of $i$ variables, $(x, y, \cdots)$ is a fixed point in $E$, and $n, m, \cdots$ are $j$ parameters that grow infinite simultaneously but independently. $\dagger$ In a recent memoir LebesGue $\ddagger$ has considered the same problems for the case where $i$ and $j$ are unity. The main part of the present discussion consists in the extension of his theorems so that they will apply to the more general integrals. The proofs of these theorems are long, and accordingly, in those cases where important new difficulties are not encountered by the addition of more variables, and also in certain other relatively unimportant instances, the proofs are omitted.

These integrals arise, in practice, especially in connection with the development of functions in series of normal functions, where the coefficients have the

* Presented to the Society, April 27, 1912.

$\dagger$ I. e., $n, m, \cdots$ belong to sequences which are independent except that they diverge to plus infinity at the same rate. This restriction concerning the rate is essential only to the proofs that the conditions are necessary. In all the convergence theorems of this paper, then, the conditions remain sufficient without this restriction, provided only it be understood that everywhere multiple, not iterated, limits are meant.

† Annales de la Faculté de Toulouse, ser. 3, vol. 1 (1909), pp. 25-128. Cf. also two theorems of HoBson in which the sufficient conditions of two of LeBEsGuE's theorems are extended so as to apply to the more general integrals, Proceedings of the London Mathematical Society, ser. 2, vol. 6 (1908), pp. 349 ff. 
form of the first integral, and the sum of the first $n m \cdots$ terms can be put in the form of the second. As applications of the theory, therefore, I have given necessary and sufficient conditions that the coefficients of such a series converge to zero, and have proved a theorem concerning the development of a function in double Fourier series. Finally, I have indicated some applications which may be made to the evaluation of multiple integrals by means of series. As far as I know, the best recent work on the subject of double Fourier series has been done by $\mathrm{H}_{\mathrm{ARDY}}$ * (1906). Other articles that have come to my notice are those written by Ascour $\dagger$ (1880), by Picard $\ddagger$ (1901), by Cerni $\S$ (1901), and by Vergerio \| (1911). Theorems corresponding to mine on the evaluation of definite integrals, for the case where the integrands are functions of a single variable, have been given by W. H. Young. $\rrbracket$ In a paper on Sturm-Liouville series of Normal Functions in the Theory of Integral Equations, MERCER ** has had occasion (page 124) to prove for one variable the sufficiency of $1^{\circ}$ in my eighth theorem. Of his two fundamental convergence theorems, the first may readily be deduced from the sufficient part of my Theorem 1, and the second has no close connection with any of my theorems.

$\S 1$. Conditions that the integral

$$
\int_{A} f(t, u, \cdots) \phi(t, u, \cdots ; n, m, \cdots) d A,
$$

where $A$ is any limited, measurable field in space of $i$ dimensions, $f$ and $\phi$ are defined in $A$, and $n, m, \cdots$ are $j$ parameters that grow infinite simultaneously but independently, $f t$ may exist, for sufficiently large values of $n, m, \cdots$, as an absolutely convergent $L$-integral $\ddagger \ddagger$ and approach zero for all functions $f$, of $i$ variables, belonging to one of the families specified below, may be stated in the several cases as follows:

Theorem 1: If $F$ is the family $F_{1}$ of absolutely L-integrable \$\& functions, it is

*Quarterly Journal of Mathematics, vol. 37 (1906), p. 53.

†'Attidella Reale Accademia dei Lincei, Memorie, ser. 3, vol. 8 (1880), pp. 263-319.

† Traité d'Analyse, 2d ed. (1901), p. 294.

8 Reale Istituto Lombardo, Rendiconti, ser. 2, vol. 34 (1901), p. 921.

\|Giornale di Matematiche, vol. 49 (1911), pp. 181-206.

IProceedings of the London Mathematical Society, ser. 2, vol. 9 (1910), p. 464. $111 \mathrm{ff}$.

* Transactions of the Royal Society of London, A, vol. 211 (1911), pp.

t+ After obvious verbal changes, these theorems are valid also in case the parameters converge to a fixed point $\left(n_{0}, m_{0}, \cdots\right)$ simultaneously but independently. See also the first footnote to this paper.

$¥ ¥$ I. e., integral in the sense of LeBesarv.

85 It should be noted that the conditions automatically require $\phi$ to be absolutely $L$-integrable, when the parameters are large, except in Theorem 6, where this requirement is separately stated. 
necessary and sufficient that $\left(1^{\circ}\right) M$ exist so that $|\phi|$ be less than $M$ when* $n, m, \cdots>n_{M}$, except perhaps at a null set of points; and that either $\left(2^{\circ}\right)$ the integral of $\phi$ over each measurable set $a$ in $A$, or else $\left(2 a^{\circ}\right)$ the integral of $\phi$ over each standard cube $\nmid q$, approch zero as $n$ becomes infinite.

CoROLlaRY: If $F$ is the family $F_{1}^{\prime}$ of L-integrable functions having only a finite number of values in $A$, it is necessary and sufficient that $2^{\circ}$ of the theorem be satisfied.

Theorem 2: If $F$ is the family $F_{2}$ of functions whose squares are L-integrable in $A$, it is necessary and sufficient that $\left(1^{\circ}\right) M$ exist so that the integral over $A$ of $\phi^{2}$ be less than $M$, when $n, m, \cdots>n_{M}$, and that either $2^{\circ}$ or else $2 a^{\circ}$ of Theorem 1 be satisfied.

Theorem 3: If $F$ is the family $F_{3}$ of functions which are limited and $L$-integrable in $A$, it is necessary and sufficient that $\left(1^{\circ}\right) M$ exist so that the integral over $A$ of the absolute value of $\phi$ be less than $M$ when $n, m, \cdots>n_{M}$, and that $2^{\circ}$ of Theorem 1 be satisfied; it is also necessary and sufficient that $\left(1 a^{\circ}\right)$ there exist for an arbitrary $\gamma>0$ two numbers, $\lambda_{\gamma}>0$, and $n_{\gamma}$, so that for every meas? set $b$ in $A$ whose measure is less than $\lambda_{\gamma}$, the integral over $b$ of the absolute value of $\phi$ is less than $\gamma$ when $n, m, \cdots>n_{\gamma}$, and that $2 a^{\circ}$ of Theorem 1 be satisfied.

COROLLARY 1: The meaning of condition $1 a^{\circ}$ is not changed if either of the following substitutions be made. (1) The integral of the absolute value of $\phi$ may be replaced by the absolute value of the integral, $\ddagger$ or (2) $b$ may be replaced by an infinite set of non-overlapping, $\S$ standard cubes $\left\{q_{i}\right\}$.

Corollary 2: For the truth of the conclusion, $1 a^{\circ}$ and $2^{\circ}$ are likewise necessary and sufficient; but $1^{\circ}$ and $2 a^{\circ}$ are necessary and not sufficient.

COROLLARY 3: If $\phi$ does not change sign in each of a finite number of measurable sets whose sum is $A, 2^{\circ}$ is necessary and sufficient.

Theorem 4: If $\boldsymbol{F}$ is the family $F_{4}$ of " simply discontinuous functions" (defined below $\$ 3$ ) in the limited, dense, metric $\|$ set $C$, it is necessary and sufficient that $\left(1^{\circ}\right) M$ exist so that the integral over $C$ of the absolute value of $\phi$ be less than $M$ when $n, m, \cdots>n_{M}$, and that $\left(2^{\circ}\right)$ the integral of $\phi$ over each dense, metric set $c$ in $C$ approach zero as $n$ becomes infinite.

CoRollary: If $F$ is the family $F_{4}^{\prime}$, a partial family of $F_{4}$ defined by means of a particular, previously chosen division of $C: C_{1}+\cdots+C_{\mu}$ (see §3), it is sufficient

* I use $n_{M}$ in place of the customary $n_{0}$ to denote a fixed number. In the proof of the necessary part of this theorem I need to use a sequence of $M$ 's and a corresponding sequence of $n_{M}$ 's. Similar reasons hold for analogous notations in other theorems.

$\dagger$ I. e., a cube in space of $i$ dimensions whose edges are parallel to the coördinate axes. Since $\phi$ is defined only in $A$, the integral is taken over only those points of $q$ that are in $A$.

$\ddagger$ In this form the condition is that the integral of $\phi$ over $A$ be "equi-absolutely continuous" in the sense of Vitali (R e ndi c onti d i Palermo, vol. 23 (1907), p. 139).

\&xcept perhaps in their boundary surfaces.

|| I. e., having content in the sense of Cantor, cf. Pierpont, Theory of Functions of Real Variables, vol. 2, p. 1. 
that $1^{\circ}$ hold, and that $\left(2 a^{\circ}\right)$ for each $C_{i} 2 a^{\circ}$ of Theorem 1 be satisfied, the integral being taken over only that part of $q$ which is in $C_{i}(i=1, \cdots, \mu)$

Theorem 5: If $F$ is the family $F_{5}$ of continuous functions of two varrables in the region $D$ (defined below, $\S 4$ ), it is sufficient that $\left(1^{\circ}\right) M$ exist so that the integral over $D$ of the absolute value of $\phi$ be less than $M$ when $n, m, \cdots>n_{\mathcal{K}}$, and that $\left(2^{\circ}\right)$ the integral over $D$ of $\phi$ approach zero as $n$ becomes infinite, and that $\left(3^{\circ}\right)$ for each $e$ the integral over $e$ of $(t \cos \alpha+u \sin \alpha-p) \phi$ approach zero as $n$ becomes infinite, where $e, \alpha$, and $p$ are as defined below ${ }^{*}(\$ 4)$. It is necessary that the same conditions be satisfied, except that the region $e$ is to be replaced by the region d (defined below, §4).

THEOREM 6: If $F$ is the family $F_{6}$ of functions " monotone increasing with respect to the point sets $\left\{B_{\lambda}\right\}$ " (defined below, $\S 5$ ), it is sufficient that $\phi$ be absolutely L-integrable, and that $\left(1^{\circ}\right)$ the integral of $\phi$ over $B_{\lambda}$ approach zero uniformly for all $\lambda$ 's as $n$ becomes infinite. It is necessary that $\left(2^{\circ}\right) M$ exist so that, if $n, m, \cdots>n_{M}, \phi$ is absolutely L-integrable and the absolute value of its integral over $B_{\lambda}$ is less than $M$ for all $\lambda^{\prime}$ 's uniformly, and that $\left(3^{\circ}\right)$ for each value of $\lambda$ this integral approach zero as $n$ becomes infinite.

CoRollary 1: If $F$ is the family $F_{6}^{\prime}$ of functions of two variables of limited variation in the sense of Hardy $\dagger$, it is sufficient that $1^{\circ}$ be satisfied uniformly for all $B_{\lambda}$ 's, when $B_{\lambda}$ and $A$ are "simple domains containing the origin, and the coördinates of each point of $A$ are positive or zero." $\ddagger$

CoRollary 2: If $F$ is the family $F_{6}^{\prime \prime}$ of functions of two variables having limited variation in the sense of PIERPONT $\S$ the conditions $2^{\circ}$ and $3^{\circ}$, with reference to each set of " monotone increasing sets $\left\{B_{\lambda}\right\}$," are necessary, $B_{\lambda}$ and $A$ being as in Corollary 1.

It is to be noted that if a function of two variables has limited variation in the sense of Hardy it may be expressed as the difference of two functions monotone increasing $\|$ with respect to the coördinates, but that not all functions which may be so expressed have limited variation. On the other hand, not all functions that have limited variation in the sense of Pierpont may be expressed as the difference of two such functions, but all functions that are so expressible do have limited variation. Example 1. The function which equals zero in that half of the square $(0 \overline{\bar{\alpha}} x, y \overline{\overline{<}} 1)$ that lies between the origin and the line, $x+y=1$, and equals unity in the remainder, is monotone in-

* These conditions are less severe than those of Theorem 4, even when $C$ is replaced by $D$.

† HARDY. Quarterly Journal of Mathematics, loc. cit., p. 58. Cf. also LEBesgue, An nales de l'École Normale, ser. 3, vol. 27 (1910), p. 408.

$\ddagger$ In the paper mentioned in the preceding note (p. 445) LEBEsGue defines such a domain as one having the property that, if the point $P$ belongs to it, so does the rectangle formed by the coördinate axes and perpendiculars to them through $P$.

\& Pierpont, loc. cit., vol. 1 (1903), p. 518.

\| Cf. HARDY, loc. cit., p. 58 (14), and p. 59 (i). Functions of limited variation, as HARDY defines them, are limited and are expressible as the difference of two limited monotone increasing functions. 
creasing and has limited variation in the sense of Pierpont, but not in the sense of Hardy. Example 2. The function which is equal to unity in the circle, $x^{2}+y^{2}=1$, and to zero elsewhere in a square enclosing this circle is not the difference of two limited monotone increasing functions, and consequently does not have limited variation in the sense of Hardy, but it does have limited variation in the sense of Pierpont.

\section{Proofs of THE PReceding theorems.}

\$2. Proofs of Theorems 1 and 2.-The proofs of these theorems for the conditions $1^{\circ}$ and $2^{\circ}$ are similar to those given by Lebesgue for the one-dimensional case. The substitution of $2^{\circ}$ for Lebesgue's $2 a^{\circ}$ simplifies the work, especially if the corollary of Theorem 1 be established first. To show the necessity and sufficiency of the other conditions the following lemmas will be used.

LEMMA * 1: If each of two functions is absolutely L-integrable in the limited, measurable field $A$, and one of them is limited, except perhaps in a null set, their product is also absolutely L-integrable in $A$.

LEMм 2: If $1 a^{\circ}$ of Theorem 3 is satisfied, then $1^{\circ}$ is also satisfied.

Suppose $\gamma=1$. By hypothesis $\lambda_{1}>0$ and therefore $\dagger \bar{A} / \lambda_{1}<\infty$. Set $M=2 \bar{A} / \lambda_{1}$, and let $A=A_{1}+\cdots+A_{p}$, where $\frac{1}{2} \lambda_{1}<\bar{A}_{i}<\lambda_{1}, i=1,2$, $\cdots, p$. Then $\bar{A}>\frac{1}{2} p \lambda_{1}$, or $p<2 \bar{A} / \lambda_{1}=M$. By hypothesis there exists an $n_{1}$ so that

$$
\begin{array}{r}
\int_{A_{i}}|\phi|<1 \quad\left(n, m, \cdots>n_{1}, i=1, \cdots, p\right) . \\
\therefore \int_{\Delta}|\phi|=\sum_{1}^{p} \int_{\Delta_{i}}|\phi|<p<M \quad\left(n, m, \cdots>n_{1}\right) .
\end{array}
$$

Lemma 3: If $1 a^{\circ}$ and $2 a^{\circ}$ of Theorem 3 are satisfied, then $1^{\circ}$ and $2^{\circ}$ are satisfied, but $1^{\circ}$ and $2 a^{\circ}$ are not sufficient to ensure $2^{\circ}$.

By the preceding lemma, $1^{\circ}$ is satisfied if $1 a^{\circ}$ is. To establish $2^{\circ}$, let $\gamma$ be greater than zero and arbitrary, and let $a$ be an arbitrary, measurable set in $A$, and enclose $a$ in a set of non-overlapping, standard cubes, $q_{1}, q_{2}, \cdots$, and suppose $p$, depending on $\lambda_{\gamma}$ and $a$, to be so chosen that

$$
\bar{q}_{1}+\cdots+\bar{q}_{p}+\bar{q}_{p+1}+\cdots-\bar{a}<\lambda_{y},
$$

$$
\bar{q}_{p+1}+\cdots<\lambda_{\gamma} .
$$

*Annales de l'École Normale, loc. cit., p. 374, $\$ 13$. In the beginning of this article will be found the principal propertics of "measure" and of multiple $L$-integrals used in the present paper. For a more complete treatment the reader should consult Pirrpont, loc. cit., vol. 2.

† Let "measure of $u$ " be denoted by $\bar{u}$. See preceding note. 
Then, by (2) and $1 a^{\circ}$, and by (1) and $1 a^{\circ}$,

$$
\begin{aligned}
& \left|\int_{\substack{\sum_{i} \\
q_{i}}} \phi-\int_{\sum_{1}^{p} q_{i}} \phi\right|=\left|\int_{\sum_{p+1}^{\infty} \phi} \phi\right|<\gamma \quad\left(n, m, \cdots>n_{\gamma}\right), \\
& \left|\int_{a} \phi-\int_{\sum_{1}^{\infty} q_{i}} \phi\right|=\left|\int_{\sum_{1}^{\infty} q_{i}-a} \phi\right|<\gamma \quad\left(n, m, \cdots>n_{\gamma}\right) .
\end{aligned}
$$

Adding (3) and (4), and using $2 a^{\circ}$, vir..,*

we have

$$
\left|\int_{\substack{p \\ \sum}} \phi\right|<\gamma \quad\left(n, m, \cdots>n_{p, \gamma}=N_{\gamma, a}\right),
$$

$$
\left|\int_{a} \phi\right|<3 \gamma \quad\left(n, m, \cdots>n_{\gamma}, N_{\gamma, a}\right) .
$$

An example of a function satisfying $1^{\circ}$ and $2 a^{\circ}$ but not $2^{\circ}$ is given below. $\dagger$

LEMMA 4: If $1^{\circ}$ of Theorem 2 is satisfied, so is $1 a^{\circ}$ of Theorem 3.

For, for any $\gamma>0$ we may select $\lambda_{\gamma}>0$ so that $\sqrt{\lambda_{\gamma} M}<\gamma$, and then, if $\bar{b}<\lambda_{\gamma}$ and $b$ is in $A$, by the inequality of Schwarz, $\ddagger$

$$
\left|\int_{b} \phi\right|<\sqrt{\int_{b} \phi^{2} \int_{b}} 1<\sqrt{M \bar{b}}<\sqrt{M \lambda_{\gamma}}<\gamma . \quad\left(n, m, \cdots>n_{x}\right) .
$$

Now, in Theorem 2 , the necessity of $2 a^{\circ}$ follows from the necessity of $2^{\circ}$. $1^{\circ}$ and $2 a^{\circ}$ are sufficient because, from them, by Lemmas 4 and $3,2^{\circ}$ follows, and $1^{\circ}$ and $2^{\circ}$ are already known to be sufficient.

Similar considerations complete the proof of Theorem 1 , for evidently, if $1^{\circ}$ of Theorem 1 is satisfied, so is $1^{\circ}$ of Theorem 2.

Proof of Theorem 3.-Lebesgue's method suffices to show that $1 a^{\circ}, 2^{\circ}$, and $2 a^{\circ}$ are necessary. From Lemma 2 it then follows that $1^{\circ}$ is necessary. By Lemma 3, then, all that remains to show in order to establish the theorem is that $1^{\circ}$ and $2^{\circ}$ are sufficient.

Let $-F<f<F$, and let the interval $(-F, F)$ be divided by the points, $a_{0}=-F, a_{1}, a_{2}, \cdots, a_{p}=F$, so that $a_{i+1}-a_{i}<\epsilon / 2 M, i=0, \cdots,(p-1)$. For each of these values of $i$ let $e_{i}$ be the set of points in $A$ where $a_{i} \bar{\Sigma} f<a_{i+1}$, and let $g$ be the function which equals $a_{i}$ in each $e_{i}$. Then, by Lemma 1 ,

$$
\left|\int_{\Delta} f \phi-\int_{\Delta} g \phi\right|=\left|\int_{\Delta}(f-g) \phi\right|<\frac{\epsilon}{2 M} \int_{\Delta}|\phi|<\frac{\epsilon}{2},
$$

* $n_{p, \gamma}$ is a fixed number after $p$ and $\gamma$ are chosen, and $p$ depends only on $\gamma$ and $a$.

$\dagger$ In the proof of Corollary 2.

† Annales de l'́́cole Normale, loc. cit., p. 442. 
if $n, m, \cdots>n_{M}$. But, by the corollary to Theorem 1 , and by $2^{\circ}$,

$$
\left|\int_{A} g \phi\right|<\frac{\epsilon}{2} . \quad\left(n, m, \cdots>n_{p, e}\right) .
$$

By the addition of (1) and (2) the theorem is now established.

Proof of Corollary 1 (Theorem 3). - Since the absolute value of the integral of a function is at most equal to the integral of its absolute value, it is evident that all that requires proof in (1) is that, assuming that for each $\gamma>0$ there exist $\lambda_{\gamma}>0$ and $n_{\gamma}$ so that the absolute value of the integral over $b$ of $\phi$ is less than $\gamma$ if $\bar{b}<\lambda_{\gamma}$, and $n, m, \cdots>n_{\gamma}$, then $1 a^{\circ}$ follows.

To prove this, having fixed $n, m, \cdots>n_{y}$, we may divide $b$ into two parts $b^{\prime}$ and $b^{\prime \prime}$, so that $\phi$ is nowhere negative in $b^{\prime}$, and everywhere negative in $b^{\prime \prime}$. Both $b^{\prime}$ and $b^{\prime \prime}$ are now special cases of $b$.

(2) is readily established, since, if $\bar{b}<\frac{1}{2} \lambda_{y}$, there exists a set of non-overlapping, ${ }^{*}$ standard cubes $\left\{q_{i}\right\}$, enclosing $b$, so that

$$
\bar{b} \bar{\gtrless} \frac{\lambda_{\gamma}}{2} \bar{\gtrless} \sum_{1}^{\infty} \bar{q}_{i}<\lambda_{\gamma} \text {. }
$$

Proof of Corollary 2 (Theorem 3).-The following function satisfies $1^{\circ}$ and $2 a^{\circ}$, and not $2^{\circ}$.

Let $A=(0,1),\{n\}=1,2,2^{2}, \cdots$. Divide the interval $(0,1)$ by the points $0,1, \frac{1}{2},\left(\frac{1}{2}\right)^{2},\left(\frac{1}{2}\right)^{3}, \cdots$, and let $\delta_{i}=\left[\left(\frac{1}{2}\right)^{i+1},\left(\frac{1}{2}\right)^{i}\right], i=0,1, \cdots$. Divide each $\delta_{i}$ into two equal intervals. $\delta_{i}^{\prime}$ and $\delta_{i}^{\prime \prime}$. Let

$$
\begin{aligned}
\phi(t, n) & =n \text { in each } \delta_{i}^{\prime}, 0 \overline{\bar{k}} t \overline{\bar{k}} 1 / n, \\
& =-n \text { in each } \delta_{i}^{\prime \prime}, 0 \overline{\bar{k}} t \overline{\bar{k}} 1 / n, \\
& =0 \text { at } t=0, \text { and where } t>1 ! n .
\end{aligned}
$$

This is the function desired. It shows that $1^{\circ}$ and $2 a^{\circ}$ are not sufficient. The rest of the corollary follows from Lemma 2 and the theorem.

§3. Explanation and Proof of Theorem 4.-We shall say that $f$ is " uniformly continuous" in a limited, metric, $\dagger$ dense set $C$, if it is defined in $C$, and for each $\epsilon>0$ there exists a finite set of non-overlapping, $\ddagger$ standard cubes, $q_{1}, q_{2}, \cdots, q_{p}$, enclosing $C$, so that $\S$ in each $q$ osf $<\epsilon$. It is known that this condition is satisfied if, in particular, $C$ is perfect, and $f$ is continuous in $C$.

* Except perhaps in their bounding surfaces.

† See footnote to Theorem 4, 81 ,

$\ddagger$ Except perhaps in their bounding surfaces.

" Osf" is an abbreviation for "oscillation of $f$," i. e., $\max f-\min f$. I use the terms maximum and minimum in the sense of upper and lower limits, not of greatest and least values. In the proof, after the first clause and until equation (4) is reacher, $f$ refers only to those values of $f$ in $C_{i}$. 
We shall say that $f$ is " simply discontinuous" in the same set $C$ if it is defined in $C$ and there exists a division of $C$ into a finite number of metric, dense parts so that in each part $f$ is uniformly continuous.

Sufficiency.-For a chosen $f$, let $C_{i}$ be one of these metric, dense parts of $C$, and let $C_{i}$ be enclosed in $q_{1}, \cdots, q_{p}$, so that in $q_{j}$

$$
\text { osf }<\epsilon
$$

$$
(j=1, \cdots p) \text {. }
$$

Let $g=k_{j}=\min f$ in $q_{j}(j=1, \cdots, p)$. Then by $2^{\circ}$, the integral over $q_{j}$ having reference only to that part of $\eta_{j}$ which is in $C_{i}$,

$$
\int_{C_{i}} g \phi=\int_{q_{1}} g \phi+\cdots+\int_{q_{p}} g \phi=k_{1} \int_{q_{1}} \phi+\cdots+k_{p} \int_{q_{p}} \phi,
$$

and approaches zero as $n$ becomes infinite. By (1) and $1^{\circ}$.

(3) $\left|\int_{C_{i}} f \phi-\int_{C_{i}} g \phi\right|=\left|\int_{C_{i}}(f-g) \phi\right| \bar{\epsilon} \epsilon \int_{C_{i}}|\phi|<\epsilon M \quad\left(n, m, \cdots>n_{M}\right)$.

From (2)

$$
\left|\int_{G} g \phi\right|<\epsilon, \quad\left(n, m, \cdots>n_{\epsilon}\right)
$$

and this added to (3) shows that the integral over $C_{i}$ of $f \phi$ approaches zero as $n$ becomes infinite. Hence, since the number of $C$ 's is finite, and since

$$
\Sigma \int_{C_{i}} f \phi=\int_{c} f \phi
$$

the last integral also approaches zero.

It is apparent that this proof applies to the corollary.

Necessity. $-2^{\circ}$ is evidently necessary, for, if a $c$ exists for which $2^{\circ}$ is not satisfied, the theorem is contradicted by the function which equals unity in $c$ and rero elsewhere.

I will now show that if $1^{\circ}$ is not satisfied there exists a continuous function, which also vanishes on the boundary of $C$, contradicting the theorem.

LemMa 1: Let $Q$ be a finite set of non-overlapping,* standard cubes, and let $\psi(t, u, \cdots)$ be defined in $Q$, and, for some $h>0$, let the integral over $Q$ of the absolute value of $\psi$ be greater than $h$. Then there exists in $Q$ a continuous function, $p(t, u, \cdots)$, which is numerically $\bar{\Sigma} 1$, and which vanishes on the boundary of $Q$, such that the integral over $Q$ of $p \psi$ is greater than $h$.

This may be shown by a method used by Lebesgue in his proof of the theorem corresponding to Theorem $\uparrow 4$.

\footnotetext{
* Except perhaps in their bounding surfaces.

† Loc. cit., p. 61.
}

Trans. Am. Math. Soc. 4 
Lеммх 2: If, in the hypothesis of Lemma 1, $C$ be substituted for $Q$, then there exists in $C$ a continuous function $g$, vanishing on the boundary of $C$, so that the integral over $C$ of $g \psi$ is greater than $h$, and $|g| \bar{\Sigma} 1$.

By hypothesis there exists a $\gamma>0$ so that

$$
\int_{C}|\psi|>h+\gamma
$$

Since by hypothesis $C$ cannot have content zero, for an arbitrary $\zeta>0$ there exists a $Q$ set of cubes, all points of which are in $C$, so that

$$
0<\bar{C}-\bar{Q}<\zeta \text {. }
$$

Choosing $\zeta>0$ so that, by a known theorem,*

$$
\int_{b}|\psi|<\gamma, \quad \text { if } \quad \bar{b}<\zeta,
$$

we have, by (2) and (3),

Hence, by (1),

$$
0 \sum \int_{c}|\psi|-\int_{Q}|\psi|=\int_{c-Q}|\psi|<\gamma .
$$

$$
\int_{Q}|\psi|>h \text {. }
$$

Now, using Lemma 1 , let $g$ be equal to $p$ in $Q$ and to zero in the rest of $C$. Then

$$
\int_{c} g \psi=\int_{Q} p \psi>h,
$$

and, since $p$ vanishes on the boundary of $Q, g$ satisfies the requirements of the lemma.

We continue now the proof of the theorem. If $1^{\circ}$ is not satisfied; for each $N_{0}$, and each $h_{0}>0$, there exist $n_{0}, m_{0}, \cdots>N_{0}$ so that

$$
\int_{c}\left|\phi\left(n_{0}, m_{0}, \cdots\right)\right|>h_{0} \text {. }
$$

Then by Lemma 2 there exists in $C$ a continuous function $g_{0}$, vanishing on the boundary of $C$, and numerically $\overline{<} 1$, so that

$$
\int_{c} g_{0} \phi\left(n_{0}, m_{0}, \cdots\right)>h_{0} \text {. }
$$

We may now proceed without difficulty in the manner outlined by Lebesgue. $\dagger$

* Annales de l'École Normale, loc. cit., p. 374.

†Annales de la Faculté de Toulouse, loc. cit., p. 61. 
§4. Explanation and Proof of Thenrem 5.-We make the following definitions. $D$ is a complete, metric * region in the $t u$-plane containing a point $O$ such that every straight line through $O$ cuts the closed curve forming the boundary of $D$ in exactly two points. There shall exist a fixed number which is greater than the number of intersections of the boundary of $D$ with any straight line. That portion of $D$ which lies on the opposite side from $O$ of any straight line is denoted by $d$; it includes its boundary; if the line passes through $O$, each portion is denoted by $d$. The equation of this striaght line shall be: $t \cos \alpha+u \sin \alpha=p$. That portion of $d$ which lies between two radial lines, including at $O$ an acute angle, is denoted by $e ; e$ also includes its boundary.

We begin by showing that the surface $f$ may be approximated by a continuous surface $F$ made up of plane facets. Let $\epsilon>0$ and $\delta>0$ be such that, in any region which can be enclosed in a circle of diameter less than $\delta$, osf $<\epsilon$. Let $D$ be enclosed in a regular poiygon $Q$, whose

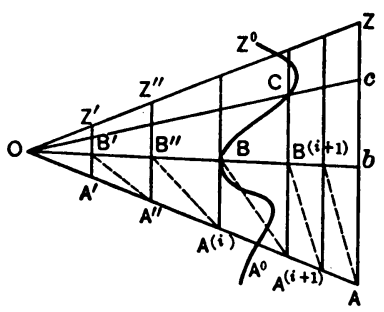

Fig. 1.

center is $O$ and whose sides have a length $<\frac{1}{2} \delta$, and let $Q$ be subdivided in the following manner. $A Z$ being any side, triangle $A Z O$ is partitioned by parallel lines, $A^{\prime} Z^{\prime}, A^{\prime \prime} Z^{\prime \prime}, \cdots, A Z$, into parts so small that each can be enclosed in a circle of diameter less than $\delta$. Each of the finite number of sides of all these parts is cut by the boundary of $D$ at most a finite number of times, so that there are between $O A$ and $O Z$ a finite number of points of intersection, $A^{\circ}, B, C, \cdots, Z^{\circ} ; A^{\circ}$ and $Z^{\circ}$ lying on $O A$ and $O Z$ respectively, and perhaps coinciding with some points already named. Draw the lines $O B b$, $O C c, \cdots ; b, c, \cdots$ heing in $A Z$; and fix the attention on $S_{1}$, the part of $D$ in $A O b$. Let $B^{\prime}, B^{\prime \prime}, \cdots$ be the intersections of $O b$ and $A^{\prime} Z^{\prime}, A^{\prime \prime} Z^{\prime \prime}, \cdots$. For some $i, O A^{(i)} \bar{O} O A^{\circ} \bar{\gamma} O A^{(i+1)}$. By construction, as one goes along the boundary of $D$ from $A^{\circ}$, the next point of irtersection $B$ with the network is on $O b$ and also on a line parallel to $A Z$. This point of intersection will therefore coincide with $B^{(i-1)}, B^{(i)}, B^{(i+1)}$, or $B^{(i+2)}$. Without loss of generality, as will become apparent, we may suppose that $B^{(i)}=B$, and that $D$ contains $A^{(i)} O B$. Draw $A^{\prime \prime} B^{\prime}, A^{\prime \prime \prime} B^{\prime \prime}, \cdots, A^{(i+1)} B^{(i)}$. Let $\tau_{1}$ be $O A^{\prime} B^{\prime}, \tau_{2}$ be $A^{\prime} A^{\prime \prime} B^{\prime}, \cdots, \tau_{k}$ be the part of $S_{\mathrm{i}}$ in $A^{(i+1)} A^{(i)} B^{(i)}$, and $\tau_{l}$ the part in

* See note to Theorem 4, 81 . 
$A^{(i+1)} B^{(i)} B^{(i+1)}$. The essential characteristics of each $\tau$ are that in it

$$
\text { osf }<\epsilon,
$$

and, except in the case of $\tau_{l}$, and perhaps of $\tau_{k}$, the three vertices are points of $D$. Let $y\left(B^{(i+1)}\right)$ be a point whose projection on the $t u$-plane is $B^{(i+1)}$ and whose distance from it equals the distance of $f\left(B^{(i)}\right)$. If $A^{(i+1)}$ does not belong to $D$, let $y\left(A^{(i+1)}\right)$ be a point whose projection is $A^{(i+1)}$ and whose distance equals the distance of $f\left(A^{(i)}\right)$. Through the points of the $f$ surface, $f(O), f\left(A^{\prime}\right), f\left(B^{\prime}\right)$, pass a plane $P_{1}$, and let the part whose projection is $\tau_{1}$ be $\pi_{1}$. Proceeding in a similar manner with the other $\tau$ 's (using the $y$ 's just defined at vertices where $f$ is not defined), and continuing the process over all the other sectors, $S_{2}, S_{3}, \cdots$, we shall have finally the surface $F$ of facets desired; for, since the distance of every other point of any facet from the $t u$-plane lies between the greatest and least distances of its vertices, by (1)

We will now show that

$$
|F-f|<\epsilon \text {. }
$$

$$
F=c+\sum_{1}^{r} f_{i}
$$

where $f_{i}=a_{i}\left(t \cos \alpha_{i}+u \sin \alpha_{i}-p_{i}\right)$ in $e_{i}, f_{i}=0$ in $D-e_{i}, c=f(0)$ $=F(0)$, a constant, and $\alpha_{i}, p_{i}$, and $e_{i}$ satisfy the definitions of $\alpha, p$, and $e$ of the hypothesis. Let the equation of the plane $P_{i}$ be $z=P_{i}(t, u)$, since no facet is perpendicular to the $t u$-plane, and let $f_{1}=P_{1}(t, u)-c$ in $S_{1}$, $=0$ in $D-S_{1}$. The line in which the plane, $z=P_{1}(t, u)-c$, cuts the $t u$-plane (since no facet need be parallel to the $t u$-plane ${ }^{*}$ ) passes through $O$, and may therefore be written $t \cos \alpha_{1}+u \sin \alpha_{1}=0$. The equation of $P_{1}$, is therefore, $z=a_{1}\left(t \cos \alpha_{1}+u \sin \alpha_{1}\right)+c$, and we may write:

$$
\begin{aligned}
f_{1} & =a_{1}\left(t \cos \alpha_{1}+u \sin \cdot \alpha_{1}\right) \text { in }\left(e_{1}=S_{1}\right), \\
& =0 \text { in } D-e_{1} . \\
z & =c+f_{1} \text { is } P_{1} \text { in } e_{1}, \\
& \text { is } \pi_{1} \text { in } \tau_{1}, \\
& \text { is } z=c \text { in } D-S_{1} .
\end{aligned}
$$

Plane $P_{2}$ intersects $P_{1}$ in a line whose projection is $A^{\prime} B^{\prime}$. Let $f_{2}=P_{2}(t, u)$ $-P_{1}(t, u)$ in $\left(e_{2}=S_{1}-\tau_{1}\right),=0$ in $D-e_{2}$. Evidently $z=P_{2}-P_{1}$ is the equation of a plane which intersects the tu-plane where $P_{2}=P_{1}$, that is, in the line of intersection $A^{\prime} B^{\prime}$. Letting the equation of $A^{\prime} B^{\prime}$ be

\footnotetext{
*If a facet were, it could be replaced by two facets that would not be.
} 
$t \cos \alpha_{2}+u \sin \alpha_{2}=p_{2}$, we may write:

$$
\begin{aligned}
f_{2}= & a_{2}\left(t \cos \alpha_{2}+u \sin \alpha_{2}-p_{2}\right) \text { in }\left(e_{2}=S_{1}-\tau_{1}\right), \\
=0 & \text { in } D-e_{2} \\
z=c+f_{1}+f_{2} & \text { is } z=c+f_{1} \text {, or } \pi_{1}, \text { in } \tau_{1}, \\
& \text { is } z=c+f_{1}+P_{2}-\left(c+f_{1}\right), \text { or } P_{2}, \text { in } e_{2}, \\
& \text { is } \pi_{2} \text { in } \tau_{2}, \\
& \text { is } z=c \text { in } D-S_{1} .
\end{aligned}
$$

Similarly $f_{3}=P_{3}-P_{2}=a_{3}\left(t \cos \alpha_{3}+u \sin \alpha_{3}-p_{3}\right)$ in $\left(e_{3}=S_{1}-\tau_{1}-\tau_{2}\right)$,

etc.

$$
=0 \text { in } D-e_{3} \text {, }
$$

The process may evidently be continued over $S_{1}$. In dealing next, in exactly the same manner, with $S_{2}$, we leave the function $z$ unchanged in $S_{1}$, because the auxiliary functions $f_{i}$ are zero outside the sector in question. The process may, therefore, be continued till we obtain the function $z=c+\sum_{1}^{r} f_{i}$, which is $\pi_{i}$ in $\tau_{i}$, and is the function $F$ used above.

Now, since the following integrals exist,*

and since

$$
\int_{D} f \phi=\int_{D}(f-F) \phi+\int_{D} F \phi
$$

$$
\int_{D} F \phi=c \int_{D} \phi+\sum_{i=1}^{r} a_{i} \int_{e_{i}}\left(t \cos \alpha_{\imath}+u \sin \alpha_{\imath}-p_{\imath}\right) \phi,
$$

in which by $2^{\circ}$ the first term on the right-hand side approaches zero as $n$ becomes infinite, and, $r$ being fixed after $\epsilon$ is chosen, the second term approaches zero by $3^{\circ}$, it follows from $1^{\circ},(2)$, and (6) that

$$
\left|\int_{D} f \phi\right|<\epsilon \int_{D}|\phi|+\left|\int_{D} F_{\phi}\right|<\epsilon M+\epsilon \quad\left(n, m, \cdots>n_{\epsilon, \mu}\right) .
$$

Necessity.-By referring to the proof of the necessary part of Theorem 4, it will be seen that $1^{\circ}$ is necessary. If $2^{\circ}$ is not satisfied, the theorem is contradicted by the function $f=1$, and if $3^{\circ}$ is not satisfied it is contradicted by the continuous function $f$ defined as follows: $f=t \cos \alpha+u \sin \alpha-p$ in $d,=0$ in $D-d$.

$\S 5$. Explanation and proof of Theorem 6 .- Let $\left\{B_{\lambda}\right\}$ be a set of " monotone increasing point sets " in the field $A$. Such a set may conveniently be defined by means of an auxiliary function $\psi(t, u, \cdots)$. Let $\psi$ be an arbitrary,

\footnotetext{
* See 82 , Lemma 1.
} 
limited, $L$-integrable function, defined in $A$. Then, for each number $\lambda, B_{\lambda}$ is the set of points where $\psi<\lambda$, and consequently $A-B_{\lambda}$ is the set where

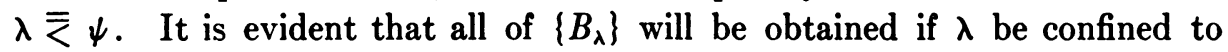
the interval * $(\min \psi, \max \psi+1)$, for, if $\lambda$ is not in this interval, $B_{\lambda}$ either consists of no points or is $A$. We shall therefore restrict $\lambda$ in this manner. Now $f$ is to be regarded as "monotone increasing with respect to $\left\{B_{\lambda}\right\}$," provided it is defined and limited in $A$, and, $\xi$ being an arbitrary number greater than $\min f$, the set of points $E_{\xi}$ where $f<\xi$ is a $B_{\lambda}$. These provisions make $f L$-integrable. $F_{6}$, then, depends for its definition on the choice of $\psi$.

Lemma: If $f(t, u, \cdots)$ belongs to $F_{6}$, and if $\phi(t, u, \cdots)$ is absolutely L-integrable in $A$,

$$
\left|\int_{\lambda} f \phi\right| \overline{<} 3 \max |f| \cdot \max _{\lambda}\left|\int_{\lambda} \phi\right| \text {. }
$$

For, by a theorem of Lebesgue's, $\nmid$ using $f, \phi$ in place of his $\phi, f$, respectively, we have

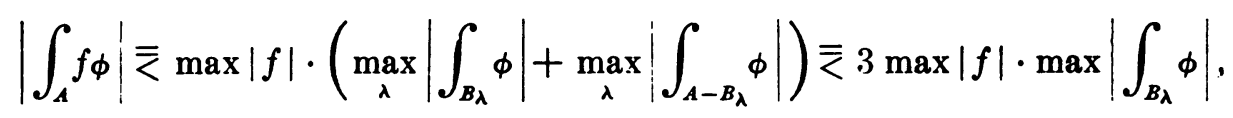

since evidently

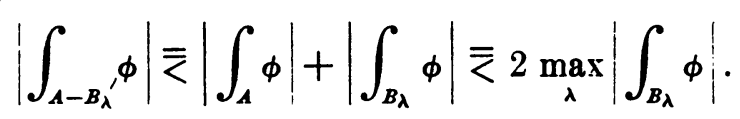

The sufficiency of condition $1^{\circ}$ follows directly from the lemma, and therefore we proceed at once to show that $2^{\circ}$ and $3^{\circ}$ are necessary.

If $\phi$ is not absolutely $L$-integrable, the function $f=1$ belongs to $F_{6}$ and the integral over $A$ of $f \phi$ does not exist as an absolutely convergent integral. If there exists a $B_{\lambda}$ for which $3^{\circ}$ is not satisfied, the function which equals -1 in this $B_{\lambda}$ and zero in $A-B_{\lambda}$ contradicts the theorem. It only remains to show that the second part of $2^{\circ}$ is necessary. To do this we shall assume a part of what has just been shown necessary, viz., that

(1) $\phi$ is absolutely $L$-integrable for each $n, m, \cdots$, and for each $\lambda, H_{\lambda}$ exists $\ddagger$ so that

$$
\left|\int_{B_{\lambda}} \phi\right|<H_{\lambda} \quad\left(n, m, \cdots>n_{H}\right) .
$$

Let us suppose $U=\left\{B_{\lambda}\right\}$ to be the set of $B_{\lambda}$ 's for which the particular family

* See the third note to $\$ 3$.

† Annales de l'Ecole Normale, loc. cit., p. 443. The notation $\max u$ is used to signify the maximum of $u$ regarded as a function of $\lambda$.

$\ddagger$ We might assume here that $H$ is arbitrarily small, i. e., $3^{\circ}$, but in the proof of Theorem 7 we need to have $2^{\circ}$ proved necessary without this assumption. 
$F_{6}$ in question exists, $U$ being defined by some preassigned function $\psi$. Suppose the second part of $2^{\circ}$ to be not satisfied. Then, for $l$ and $N$ arbitrary, there exist the numbers $n(l), m(l), \cdots>N$, and $\lambda(l)$, so that

$$
\left|\int_{B_{\lambda(l)}} \phi[n(l), m(l), \cdots]\right|>l \text {. }
$$

By (2) there exists a number $M$, which we may take $>1$, and a corresponding $n_{M}$, so that

$$
\left|\int_{\Lambda} \phi(n, m, \cdots)\right|<M \quad\left(n, m, \cdots>n_{M}\right) .
$$

Now let $l=2 M>2, N_{1}=n_{M}$. By (3) there exist the numbers $n_{1}, m_{1}, \ldots$ $>N_{1}+1$, and $\lambda_{1}$, so that

Take, next,

$$
\left|\int_{B_{\lambda_{1}}} \phi\left(n_{1}, m_{1}, \cdots\right)\right|>2 M>2 .
$$

$$
l=\left|\int_{B_{\lambda_{1}}} \phi\left(n_{1}, m_{1}, \cdots\right)\right|+1, N_{2}=\max \left(n_{1}, m_{1}, \cdots\right)>N_{1}+1 .
$$

Then there exist the numbers $n_{2}, m_{2}, \cdots>N_{2}+2$, and $\lambda_{2}$, so that

$$
\left|\int_{B_{\lambda_{2}}} \phi\left(n_{2}, m_{2}, \cdots\right)\right|>\left|\int_{B_{\lambda_{1}}} \phi\left(n_{1}, m_{1}, \cdots\right)\right|+1 .
$$

Proceeding in this manner we have finally the following sequences, of which the first and third diverge to infinity:

$$
\begin{gathered}
n_{\mathcal{M}}<n_{1}, \\
\lambda_{1},
\end{gathered}
$$$$
\text { (7) } 2<2 M<\left|\int_{B_{\lambda_{1}}} \phi\left(n_{1}, m_{1}, \cdots\right)\right|<\left|\int_{B_{\lambda_{2}}} \phi\left(n_{2}, m_{2}, \cdots\right)\right|<\cdots
$$

No $\lambda_{i}$ is repeated an infinite number of times, for otherwise the absolute value of the integral over $B_{\lambda_{4}}$ of $\phi(n, m, \cdots)$ diverges to infinity for a certain sequence of $(n, m, \cdots)$ 's, and therefore the function $f$ which equals -1 in $B_{\lambda_{4}}$ and 0 in $A-B_{\lambda_{4}}$ contradicts the theorem. Consequently infinitely many of the $\lambda_{i}$ 's are distinct, and we may assume without loss of generality that they are all distinct.

Since the $\lambda$ 's are infinite in number they may be represented by a point-set in $(\min \psi, \max \psi+1)$ which must have at least one limit point, $L$; and therefore there exists among the $\lambda^{\prime}$ 's an infinite set, $\lambda^{\prime}, \lambda^{\prime \prime}, \cdots$, having $L$ as a limit point, such that, either 
(a)

$$
\lambda^{\prime}>\lambda^{\prime \prime}>\cdots,
$$

or

$$
\lambda^{\prime}<\lambda^{\prime \prime}<\cdots,
$$

or both. By the definition of $B_{\lambda}$, then, either

$$
B_{\lambda^{\prime}}>B_{\lambda^{\prime \prime}} \overline{\bar{N}} \cdots \text {, }
$$

$$
B_{\lambda^{\prime}} \overline{\bar{\prime}} B_{\lambda^{\prime \prime}} \overline{\bar{\alpha}} \cdots \text {. }
$$

Case B.-By (7) and (4), for an arbitrary $l$ and an arbitrary $N$, there exists a $\lambda^{(i)}$ of $(b)$ so that $n^{(i)}, m^{(i)}, \cdots>N$, and simultaneously

$$
\left|\int_{B_{\lambda}(i)} \phi\left(n^{(i)}, m^{(i)}, \cdots\right)\right|>2 M+l, \quad\left|\int_{\Lambda} \phi\left(n^{(i)}, m^{(i)}, \cdots\right)\right|<M .
$$

Applying these inequalities to the identity

we have

$$
\int_{A-B_{\lambda}(i)} \phi\left(n^{(i)}, m^{(i)}, \cdots\right)=\int_{\Lambda}-\int_{B_{\lambda}(i)},
$$

$$
\left|\int_{\Delta-B_{\lambda}(i)} \phi\left(n^{(i)}, m^{(i)}, \cdots\right)\right|=\left|\int_{\Delta}-\int_{B_{\lambda}(i)}\right|>-M+2 M+l=M+l .
$$

There exists in $(b)$ a sequence $\lambda^{\left(i_{1}\right)}, \lambda^{\left(i_{s}\right)}, \ldots$ so that

$$
\begin{aligned}
& \left|\int_{\Delta-B_{\lambda}\left(i_{1}\right)} \phi\left(n^{\left(i_{1}\right)}, m^{\left(i_{1}\right)}, \cdots\right)\right|<\left|\int_{\Delta-B_{\lambda}\left(i_{2}\right)} \phi\left(n^{\left(i_{2}\right)}, m^{\left(i_{2}\right)}, \cdots\right)\right|<\cdots,
\end{aligned}
$$

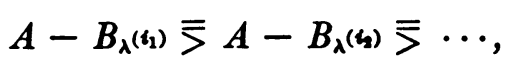

and the former sequence diverges to infinity. By reference to (7) it is now clear that, without loss of generality, we may assume Case $A$; and, to save changing subscripts, let us suppose ( $a$ ) and $(A)$ to have been true of (7). Case $B$, continued, would mean that the $g$ 's defined later would be related to $A-B_{\lambda}$ as, in Case $A$, they are related to $B_{\lambda}$, except that the numbers would be positive instead of negative. Assuming Case $A$, then, we may add to (7):

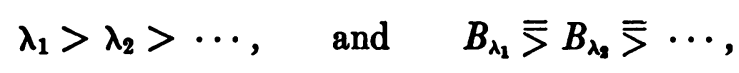

and here the $\lambda$ 's are all different and the first sequence has the limit $L$.

We are now ready to show the existence of a function $g$ which contradicts the theorem. Setting $l_{1}=2 M$, and so, rewriting

$$
\left|\int_{B_{\lambda_{1}}} \phi\left(n_{1}, m_{1}, \cdots\right)\right|>l_{1}=2 M,
$$


we note that, since, $n_{1}, m_{1}, \cdots$ are fixed, the following is, by (1) finite:

$$
L_{1}=\max _{\lambda}\left|\int_{B_{\lambda}} \phi\left(n_{1}, m_{1}, \cdots\right)\right|
$$

Let $g_{1}=0$ in $A-B_{\lambda_{1}}$, $=-1$ in $B_{\lambda_{1}}$. If the integral over $A$ of $g_{1} \phi(n, m, \cdots)$ does not approach zero as $n$ becomes infinite, $g_{1}$ is the desired function, for $B_{\lambda_{1}}$ belongs to $U$ and therefore $g_{1}$ to $F_{6}$. Supposing, the worse case then, that this is not true, there exists a $\mu_{1}>0$ so that

$$
\left|\int_{A} g_{1} \phi(n, m, \cdots)\right|<1 \quad\left(n, m, \cdots>\mu_{1}\right) .
$$

Set

$$
l_{2}=2^{2} L_{1} \text {. }
$$

By going far enough in (7) to a $\lambda$ which, dropping intermediate $\lambda$ 's, we may rename $\lambda_{2}$, we have simultaneously

$$
\left|\int_{B_{\lambda_{2}}} \phi\left(n_{2}, m_{2}, \cdots\right)\right|>2^{2} L_{1}=l_{2} ; n_{2}, m_{2}, \cdots>\mu_{1} .
$$

Set

$$
\begin{aligned}
L_{2} & =\max _{\lambda}\left|\int_{B_{\lambda}} \phi\left(n_{2}, m_{2}, \cdots\right)\right|, \\
g_{2} & =0 \quad \text { in } A-B_{\lambda_{2}}, \\
& =-\frac{2}{l_{2}}=\frac{-1}{2 L_{1}} \text { in } B_{\lambda_{2}} .
\end{aligned}
$$

By (11) and (8), respectively,

$$
L_{2}>L_{1}, \quad \text { and } \quad B_{\lambda_{1}} \bar{₹} B_{\lambda_{2}} .
$$

Consider the function $g_{1}+g_{2}$.

$$
\text { In } B_{\lambda_{2}}, g_{1}+g_{2}=-1-2 / l_{2}<-1 \text {. }
$$

In $A-B_{\lambda_{2}}, g_{1}+g_{2}=$ either -1 , or 0 .

In $B_{\lambda_{1}}, g_{1}+g_{2}=$ either $-2 / l_{2}-1$, or $-1,<0$.

In $A-B_{\lambda_{1}}, g_{1}+g_{2}=0$.

For any number $\xi$ which is greater than min $\left(g_{1}+g_{2}\right)$, the set of points where $g_{1}+g_{2}<\xi$ is either $B_{\lambda_{2}}, B_{\lambda_{1}}$, or $A$, all of which belong to $U$. Hence $g_{1}+g_{2}$ belongs to $F_{6}$. Therefore, if the integral over $A$ of the product $\left(g_{1}+g_{2}\right) \phi(n, m, \cdots)$ does not approach zero as $n$ becomes infinite, $g_{1}+g_{2}$ is the function desired.

Otherwise, there exists $\mu_{2}>0$ so that

$$
\left|\int_{\Lambda}\left(g_{1}+g_{2}\right) \phi(n, m, \cdots)\right|<1 \quad\left(n, m, \cdots>\mu_{2}\right) .
$$


Set

$$
l_{3}=2^{3} L_{2} \text {, etc. }
$$

Finally, if (the worst case) the number of steps is infinite, there exists a function $g$, limited in $A$,

$$
g(t, u, \cdots)=g_{1}+g_{2}+\cdots,
$$

where ${ }^{*} g_{i}=-2 / l_{i}$ in $B_{\lambda_{4}}$, $=0$ in $A-B_{\lambda_{4}}$; for (17) may be compared with the series,

$$
-1-\frac{2}{l_{2}}-\frac{2}{l_{3}}-\cdots=-1-\frac{1}{2 L_{1}}-\frac{1}{2^{2} L_{2}} \cdots,
$$

and this converges, since, by (14) and corresponding formulæ for the later indices, $L_{1}<L_{2}<\cdots$. Moreover, $g$ belongs to $F_{6}$; for, by (8), we have for any $i$

$$
\begin{aligned}
& \text { in } B_{\lambda_{1}}, g \bar{\Sigma} g_{1}+\cdots+g_{i}=-1-\frac{2}{l_{2}}-\cdots-\frac{2}{l_{i}}<-1-\frac{2}{l_{2}}-\cdots-\frac{2}{l_{i-1}}, \\
& \quad \text { in } A-B_{\lambda_{1}}, g=g_{1}+\cdots+g_{i}>-1-\frac{2}{l_{2}}-\cdots-\frac{2}{l_{i-1}},
\end{aligned}
$$

and, for any $\xi$ such that $\min g<\xi<\max g+1, B_{\xi}$ is some $B_{\lambda_{k}}$, which belongs to $U$. It only remains to show, then, that

$$
\int_{\Lambda} g \phi(n, m, \cdots)
$$

does not converge to zero as $n$ becomes infinite.

For each subscript $j>2$,

$$
\begin{aligned}
\int_{\Delta} g \phi\left(n_{j}, m_{j}, \cdots\right)=\sum_{i=1}^{j-1} \int_{\Delta} g_{i} \phi\left(n_{j}, m_{j}, \cdots\right)+\int_{\Delta} g_{j} \phi\left(n_{j}, m_{j}, \cdots\right) \\
\quad+\sum_{i=j+1}^{\infty} \int_{\Lambda} g_{i} \phi\left(n_{j}, m_{j}, \cdots\right)=\mathrm{I}+\mathrm{II}+\mathrm{III} .
\end{aligned}
$$

By (15) etc., $|\mathrm{I}|<1$.

By (13) etc., and by (11) etc.,

$$
|\mathrm{II}|=\left|\int_{B_{\lambda_{j}}} \frac{1}{2^{j-1} L_{j-1}} \phi\left(n_{j}, m_{j}, \cdots\right)\right|>\frac{2^{j} L_{j-1}}{2^{j-1} L_{j-1}}=2 .
$$

By successive applications of (13) etc., (12) etc., and (14) etc., we have

$$
\begin{aligned}
& |\mathrm{III}|=\left|\frac{1}{2^{j} L_{j}} \int_{B_{\lambda_{j+1}}} \phi\left(n_{j}, m_{j}, \cdots\right)+\frac{1}{2^{j+1} L_{j+1}} \int_{B_{\lambda_{j+8}}} \phi\left(n_{j}, m_{j}, \cdots\right)+\cdots\right| \\
& \bar{\sum}\left(\frac{1}{2^{j} L_{j}}+\frac{1}{2^{j+1} L_{\jmath+1}}+\cdots\right) L_{j}<\frac{1}{2^{j}}+\frac{1}{2^{j+1}}+\cdots<\frac{1}{2} \quad(j>2) .
\end{aligned}
$$

* Except that $g_{1}=-2 M / l_{1}$. 
Hence, if $j>2$,

and (18) is proved.

$$
|\mathrm{I}+\mathrm{II}+\mathrm{III}|>2-1-\frac{1}{2}=\frac{1}{2},
$$

$\S 6$. Let us now consider the integral

$$
\int_{\boldsymbol{E}} f(t, u) \theta(t, u ; x, y ; n, m) d E
$$

and its convergence to $f(x, y)$ at the arbitrary, fixed point $(x, y)$ of the limited, measurable field $E$, in space of two * dimensions.

THEOREM 7: Let (a) $f$ be continuous at $(x, y),(b) f$ belong to one of the families $F$ of the previous theorems, (c) $E$ satisfy the conditions imposed on the field of integration in that one of the preceding theorems which relates to the $F$ in question, (d) $V_{8}$ be a circle of radius $\delta>0$, whose center is $(x, y)$, and suppose also that $E$ is dense and metric in some $V_{8}$.

Conditions that this integral may approach $f(x, y)$, as $n$ becomes infinite, for all f's belonging to one of the families $F$, may be stated in the several cases as follows:

If $F$ is $F_{1}, F_{1}^{\prime}, F_{2}, F_{3}$, or $F_{4}$, it is necessary and sufficient that there exist a $\delta_{0}$ so that, for every positive $\delta<\delta_{0},\left(1^{\circ}\right)$ the conditions of the previous theorem relating to the $F$ in question are satisfied for $E-V_{\delta}$, that $\left(2^{\circ}\right)$ there exist an $M$ so that the integral over $V_{\delta_{0}}$ of the absolute value of $\theta$ is less than $M$, if $n, m>n_{\mathcal{X}}$, and that $\left(3^{\circ}\right)$ the integral over $E$ of $\theta$ approach unity as $n$ becomes infinite.

If $F$ is $F_{6}$ or $F_{6}^{\prime}$, it is sufficient that the sufficient conditions involved in $1^{\circ}$ be satisfied with respect to $B_{\lambda}^{\prime}$, where $B_{\lambda}^{\prime}$ is the part of $B_{\lambda}$ in $E-V_{\delta}$, that $3^{\circ}$ be satisfied, and that $\left(2 a^{\circ}\right)$ there exist an $M$ so that the absolute value of the integral over $B_{\lambda}^{\prime \prime}$ of $\theta$ is less than $M$, if $n, m>n_{\mathcal{M}}$, for all $\delta^{\prime} s \leqq \delta_{0}$ and all $\lambda$ 's uniformly, where $B_{\lambda}^{\prime \prime}$ is the part of $B_{\lambda}$ in $V_{\delta}$.

If $F$ is $F_{5}$, it is necessary and sufficient that the necessary and sufficient conditions, respectively, of Theorem 5 be satisfied relative to $E, 3^{\circ}$ replacing $2^{\circ}$ of Theorem 5, and the region d being defined by using $(x, y)$ as a new origin; this imposes a certain restriction on $E$ (cf. the second sentence in $\$ 4$ ).

The proof of this theorem follows from the preceding theorems in much the same manner as the proof of the corresponding theorem $\dagger$ in one dimension follows from their analogues.

\section{ApPlichtions to SERIES OF NORMal FUNCTIONS.}

\$7. ThEOREM 8: Let. $w_{i}(t, u, \cdots)$ be a complete set of normal $¥$ functions in

* The generalization to more than two dimensions in certain cases is evident from the preceding theorems. See also the first note to $\& 1$.

t Annales de la Faculté de Toulouse, loc. cit., p. 70. Note also the remark concerning the integral on page 28.

$¥$ I. e., orthogonal functions of which the integrals of the squares over the field in question are equal to unity. Consult, e. g., RiEsz, next footnote. 
the limited, measurable field $A$. In order that the coefficients of the formal development of $f(t, u, \cdots)$ in terms of the w's may exist for sufficiently large values of $i$ and approach zero as $i$ becomes infinite, for all functions $f$ of each of the families $F_{1}, F_{2}, F_{3}, F_{4}$, and $F_{5}$, previously considered, it is necessary and sufficient that in each case there exist an $M$ so that when $i>i_{M}$ the inequality mentioned below for the family in question is satisfied:

If $F$ is $F_{1},\left(1^{\circ}\right)\left|w_{i}\right|$ shall be less than $M$.

If $F$ is $F_{2},\left(2^{\circ}\right)$ the integral over $A$ of $w_{i}^{2}$ shall be less than $M$.

If $F$ is $F_{3},\left(3^{\circ}\right)$ the integral over $A$ of $\left|w_{i}\right|$ shall be less than $M$.

If $F$ is $F_{4}$, or $\left.F_{5}, 4^{\circ}\right)$ the integral over $C$ of $\left|w_{i}\right|$ shall be less than $M$.

Corollary: Similar statements may be made for functions of one variable, and in that case we have the additional statement that if $f$ is a function of limited variation, it is necessary and sufficient that $M$ exist so that, if $i>i_{M}$, the absolute value of the integral from $a$ to $\lambda$ of $w_{i}$ is less than $M$ for all $\lambda$ 's in $(a, b),(a, b)$ being the interval in which the functions are defined.

It will be shown that if $F$ is $F_{1}, 1^{\circ}$ is necessary and sufficient. If $F$ is some other family, the proof is analogous.

Sufficiency.-F. RIEsz ${ }^{*}$ has shown that if $\left\{w_{i}\right\}$ is as in our hypothesis, and if $f^{2}$ (and therefore, by ScHwarz's inequality, $\dagger f$ ) is absolutely $L$-integrable, the series of the squares of the coefficients,

$$
\sum_{i=1}^{\infty}\left[\int_{A} f w_{i}\right]^{2}
$$

is convergent. Hence, under these conditions, each coefficient, (1) $\int_{A} f w_{i}$, approaches zero as $i$ becomes infinite, and this is true in particular when $f=1$ in $a$ and zero elsewhere, $a$ being any measurable component of $A$. That is, provided only that $\left\{w_{i}\right\}$ be as above, (2) $\int_{a} w_{i}$ approaches zero as $i$ becomes infinite. This is condition $2^{\circ}$ of Theorem 1 , and in our hypothesis $1^{\circ}$ is condition $1^{\circ}$ of that theorem, $w_{i}$ being a special case of $\phi(n, m, \cdots)$. Hence (1) follows from $1^{\circ}$ and (2).

Necessity.-If $w_{i}$ does not satisfy $1^{\circ}$, then by Theorem 1 there exists an $f$ belonging to $F_{1}$ for which the integral over $A$ of $f w_{i}$ does not approach zero as $i$ becomes infinite. Therefore $1^{\circ}$ is necessary.

$\S 8$. Double Fourier Series: Let $f(t, u)$ have the double period $2 \pi$ in the $t u$-plane, and be absolutely $L$-integrable in the square $S$, where $S$ is $(x-\pi \bar{₹} t \bar{\gtrless} x+\pi, y-\pi \overline{\bar{\gamma}} u \overline{\bar{\gamma}} y+\pi)$, and $(x, y)$ is an arbitrary,

* C o m p t e s R e n d u s, vol. 144 (1907), p. 616, p. 734. Rirsz does not state that $f^{2}$ is supposed $L$-integrable, but he implies it in his proof.

† Annales de l'École Normale, loc. cit., p. 442. 
fixed point. We may formally develop $f(x, y)$ in a double series of the form,

$$
\sum_{i, j=1}^{\infty}\left[\left(a_{i j} \sin i x+b_{i j} \cos i x\right) \sin j y+\left(c_{i j} \sin i x+d_{i j} \cos i x\right) \cos j y\right] \text {. }
$$

As is well known, it may be shown in a manner analogous to the method used in simple series that the sum of all those terms whose subscripts are not greater than $n, m$, respectively, may be put in the form:

$S(n, m ; x, y)=\frac{1}{\pi^{2}} \int_{S} f(t, u)\left[\frac{\sin \frac{1}{2}(2 n+1)(t-x)}{2 \sin \frac{1}{2}(t-x)} \frac{\sin \frac{1}{2}(2 m+1)(u-y)}{2 \sin \frac{1}{2}(u-y)}\right]$.

We now define the sum of the double series to mean the double * limit of $S(n, m)$, if this limit exists, as $n, m$ become infinite. We will show that, under certain circumstances, this limit does exist and is equal to $f(x, y)$. In this integral we make the substitutions:

$$
t-x=2 \alpha^{\prime}, u-y=2 \beta^{\prime} ; 2 n+1=\nu, 2 m+1=\mu .
$$

$S$ becomes the square $\left(-\pi / 2 \bar{\gtrless} \alpha^{\prime}, \beta^{\prime} \bar{\Sigma} \pi / 2\right)$. Then, making the transformation,

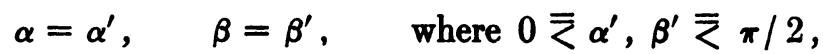

$$
\begin{aligned}
& \alpha=-\alpha^{\prime}, \quad \beta=\beta^{\prime}, \quad \text { where }-\pi / 2 \bar{k} \alpha^{\prime} \bar{\gtrless} 0 \text { and } 0 \bar{\sum} \beta^{\prime} \bar{\Sigma} \pi / 2 \text {, }
\end{aligned}
$$

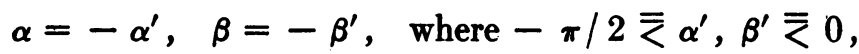

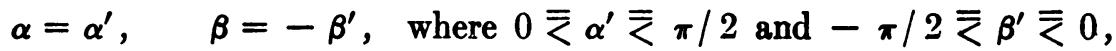

and proceeding in a manner analogous to the well-known $\dagger$ theory for simple Fourier series, we may learn that the validity of the expansion depends on the convergence to zero, as $\nu$ becomes infinite, of the integral

$$
\begin{aligned}
& D(\nu, \mu)= \int_{S_{1}} \frac{\sin \nu \alpha \sin \mu \beta}{\sin \alpha \sin \beta} \phi(\alpha, \beta), \text { where } \\
& \phi(\alpha, \beta)= f(x-2 \alpha, y-2 \beta)+f(x+2 \alpha, y-2 \beta) \\
&+f(x-2 \alpha, y+2 \beta)+f(x+2 \alpha, y+2 \beta)-4 f(x, y), \\
& \text { and } S_{1} \text { denotes the square, }(0 \overline{\bar{k}} \alpha, \beta \bar{₹} \pi / 2) .
\end{aligned}
$$

The substitutions made above are valid if the various integrals may be expressed as iterated integrals, $\ddagger$ a condition which is fulfilled since the double

* Cf. the first footnote to this paper.

† E. g., Puerpont, loc. cit., vol. 2, pp. 421-3.

† Annales de l'École Normale, loc. cit., pp. 447-50. 
integrals * exist, $f$ being absolutely $L$-integrable $\dagger$ and $\sin \nu \alpha / \sin \alpha$, $\sin \mu \beta / \sin \beta$ being limited for each $\nu, \mu$.

Theorem 9: If $f(t, u)$ is absolutely L-integrable in $S$, its double Fourier series is valid at the fixed point $(x, y)$, provided, in $(2), \phi(\alpha, \beta) / \alpha \beta$ is absolutely L-integrable in $S_{1}$.

Proof.-Writing $D(\nu, \mu)$ in the form,

$$
\int_{S_{1}} \frac{\phi(\alpha, \beta)}{\alpha \beta} \frac{\alpha \sin \nu \alpha \cdot \beta \sin \mu \beta}{\sin \alpha \sin \beta},
$$

we learn from Theorem 1 that it is sufficient to show that

$$
\left|\frac{\alpha \sin \nu \alpha \cdot \beta \sin \mu \beta}{\sin \alpha \sin \beta}\right|<\text { some } M \text {, for all } \nu, \mu \text {, }
$$

and

$$
\int_{q}^{\alpha \sin \nu \alpha \cdot \beta \sin \mu \beta} \frac{\sin \alpha \sin \beta}{\sin }
$$

approaches zero for each $q$ in $S_{1}$, as $\nu, \mu$ become infinite. Since $1 \overline{\overline{<}} \alpha / \sin \alpha \overline{\overline{2}} \pi / 2$, $\alpha$ in $(0, \pi / 2)$, and $|\sin \nu \alpha| \bar{\Sigma} 1,(3)$ is evident. We may write (4) in the forms,

$$
\int_{a}^{b} \frac{\alpha \sin \nu \alpha}{\sin \alpha} d \alpha \int_{a}^{b} \frac{\beta \sin \mu \beta}{\sin \beta} d \beta=\left[\int_{a}^{b} \frac{\alpha \sin \nu \alpha}{\sin \alpha} d \alpha\right]\left[\int_{a}^{b} \frac{\beta \sin \mu \beta}{\sin \beta} d \beta\right],
$$

since the double integral exists, and the limits, $a, b$, are constant. Now $\alpha / \sin \alpha$ is monotone increasing in $(0, \pi / 2)$, and $\sin \nu \alpha$ is $L$-integrable. Hence the lemma of $\S 5$ applies, and

$$
\begin{aligned}
& \left|\int_{a}^{b} \frac{\alpha \sin \nu \alpha}{\sin \alpha} d \alpha\right| \bar{z} \max \left|\frac{\alpha}{\sin \alpha}\right| \cdot \max _{\lambda}\left|\int_{a}^{\lambda} \sin \nu \alpha d \alpha\right| \\
& \bar{\Sigma} 3 \cdot \frac{\pi}{2} \cdot \frac{1}{\nu} \max _{\lambda}|\cos \nu a-\cos \nu \lambda| \bar{\Sigma} \frac{3 \pi}{\nu} \text {. } \\
& \left|\int_{a}^{b} \frac{\alpha \sin \nu \alpha}{\sin \alpha} d \alpha \int_{a}^{b} \frac{\beta \sin \mu \beta}{\sin \beta} d \beta\right| \overline{\frac{9 \pi^{2}}{\nu \mu}},
\end{aligned}
$$

and (4) is proved.

CoRollary: If $f$ is absolutely L-integrable in $S$, the validity of the expansion at $(x, y)$ depends only on the behavior of the function in the vicinity of the lines through this point parallel to the coördinate axes.

For it is only in this vicinity that additional restrictions on $f$ are needed in order to make $\phi / \alpha \beta$ integrable. Cf. Lemma $1, \S 2$.

* Hobson, Proceedings of the London Mathematical Society, ser. 2, vol. 8 (1910), p. 30.

† See Lemma 1, \& 2. 
Theorem 10: If $f$ is absolutely L-integrable in $S$, the coefficients of its formal series at $(x, y)$ approach zero as a double limit as $n, m$ become infinite.

For sin $n t$ and $\cos n t$ are numerically less than or equal to unity for all values of $n$ and $t$. Cf. Theorem $8,1^{\circ}$.

REMaRK: These theorems include corresponding theorems in simple Fourier series (already known) as special cases, and may be stated more generally so as to apply to multiple Fourier series of functions having any desired periods in the $i$ variables, $t, u, \cdots$.

\section{Definite Integrals.}

\$. Other applications of the fundamental theorems may be found in the evaluation of absolutely convergent, multiple $L$-integrals of the form,

$$
\int_{A} f(t, u, \cdots) g(t, u, \cdots) d A,
$$

by means of the device of expanding one of these functions, say $g$, in a multiple series, multiplying through by $f$, and integrating termwise. If the partial sum of such a series be denoted by $s_{n}, m, \ldots$, it is clear that this process is equivalent to the requirements that the integral over $A$ of the product, $f\left(g-s_{n, m}, \ldots\right)$, exist for sufficiently large values of $n, m, \cdots$, and that its multiple limit be zero. In Theorems $1-6$, therefore, replacing $\phi(n, m, \cdots)$ by $g-s_{n}, m, \ldots$, the reader will find stated the necessary and sufficient conditions that the process be allowable.

It will be noticed that condition $2^{\circ}$ is satisfied if the series for $g$ converges, except perhaps for a null set, and is integrable termwise in each set $a$. VrTali* has shown that a simple series of limited functions of one variable has this property if it converges to an integrable function, and if condition $1 a^{\circ}$ of Theorem 3 (cf. Corollary 1, Theorem 3, and footnote) is satisfied. His reasoning is sufficient, however (by the aid of a theorem of Lebesguet), to establish the same result for simple series of absolutely $L$-integrable functions of $i$ variables defined in $A$, whether these functions are limited or not. Therefore, at least if simple series be used, the conditions that the above process of evaluation be valid, whenever the $g$ series converges to $g$ (except perhaps at a null set), and all the integrals involved exist, may be stated more simply in certain cases, as follows:

If $f$ is in $F_{1}$, it is sufficient that $\left|g-s_{n}\right|$ be less than $M$, except perhaps at a null set, when $n>n_{M}$.

\footnotetext{
* Rendiconti di Palermo, vol. 23 (1907), p. 139. Vitali uses $1 a^{\circ}$ with reference to $s_{n}$ instead of $g-8_{n}$, but it is readily shown that there is no difference, since $g$ is integrable.

† Annales de l'École Normale, loc. cit., p. 375, \&15. A fundamental theorem in the theory of measurable sets is also needed. Cf. Prerpont, loc. cit., vol. 2, p. 358.
} 
If $f$ is in $F_{2}$, it is sufficient that the integral over $A$ of $\left(g-s_{n}\right)^{2}$ be less thian $M$, when $n>n_{M}$.

If $f$ is in $F_{3}$, it is sufficient that for each $\gamma>0$ there exist two numbers, $\lambda_{\gamma}>0$, and $n_{\gamma}$, so that for every measurable set $b$ in $A$ whose measure is less than $\lambda_{\gamma}$, the integral over $b$ of the absolute value of $\left(g-s_{n}\right)$ is less than $\gamma$ when $n>n_{\gamma}$. For it has previously been shown that if $1^{\circ}$ of either Theorem 1 or Theorem 2 is satisfied, so is $1 a^{\circ}$ of Theorem 3.

These conditions are also necessary in the same sense as the conditions of Theorem 1 are necessary; that is, if, for example, the first condition is not satisfied, then there exists an $f$ belonging to $F_{1}$ for which the process is not valid.

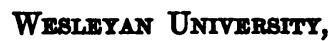
MiddLeTOWN, CONN. 